\title{
APPLICATION OF THE LATTICE BOLTZMANN METHOD TO LIQUID-SOLID PHASE CHANGE IN FREE SURFACE FLOW: AN EXAMPLE OF MONGOLIAN SMALL HYDROPOWER PLANT
}

\author{
Badarch AYURZANA ${ }^{1}$ and Tokuzo HOSOYAMADA ${ }^{2}$ \\ 1 Ph.D student, Graduate School of Eng., Nagaoka University of Technology \\ (1603-1 Kamitimioka, Nagaoka 940-2188, Japan) \\ E-mail:ayur_426@yahoo.com \\ 2 Member of JSCE, Dept. of Civil and Environmental Eng., Nagaoka University of Technology \\ (1603-1 Kamitimioka, Nagaoka 940-2188, Japan) \\ E-mail:rng@nagaokaut.ac.jp
}

\begin{abstract}
The presence of ice hinders the operation of small hydropower plants in cold regions like Mongolia. In order to study this problem, we propose a numerical model for analyzing the liquid-solid phase changes that occur in a free surface flow. This model is formulated using the lattice Boltzmann method (LBM). The single phase free surface model, along with the immersed boundary condition, is used to model the free surface flow and solid phase. The thermal lattice Boltzmann (LB) equation is combined with a non-iterative enthalpy based formulation to simulate heat transport and phase changes. These models are integrated in a way that holds physical relation. The performance and accuracy of the model is validated using experimental evaluations. The formation of open water in the downstreamchannel of the hydropower plant is successfully simulated to demonstrate the performance of the model. Results indicate that the proposed model can be used for studying measures to control downstream ice in hydropower plants. It can also be applied to study the ice phenomena in open channel flows.
\end{abstract}

Key Words : ice melting, lattice Boltzmann method, free surface flow, ice problem in hydropower plants

\section{INTRODUCTION}

Off-the-grid small hydropower plants (SHPPs) have been promoted as an efficient source to fulfill the electricity demands in the rural areas of Mongolia ${ }^{1)}$. A total of 11 SHPPs have been commissioned since $1986^{2)}$ and a number of plants have been planned for future construction. However, except for two of the plants, the others can be operated only for a short duration. The rest are unable to operate during the winter, from the time the river ice freezes until the ice breaks. In this study, we consider one of the SHPPs, namely the Tosontsengel hydropower plant, shown in Fig.1. This is located in the village of Tosontsengel, which is one of the coldest places in Mongolia. The hydropower plant operates on the river Ider, which has a flow under the ice cover during the winter ${ }^{3)}$. At this time, the plant faces a serious ice problem in the downstream area. The ice problem in the outlet channel of the turbine not only reduces energy production but also decreases the cross-sectional area of the water way, which causes bed erosion in the unlined channel.
In general, studies on the ice problem and the possible countermeasures ${ }^{4)}$ are based on on-site measurements. There are very few studies that consider the prediction or modeling of the ice problem. In physical terms, the behavior of ice in the outlet of a hydropower plant in a cold region can be modeled as an interaction between ice and the free surface flow. This involves liquid-solid phase changes since warm water is released from the turbine to the outflow channel changing the ice condition.

A novel numerical method based on the lattice Boltzmann method (LBM) is proposed in this paper. The method consists of a combination of modules,

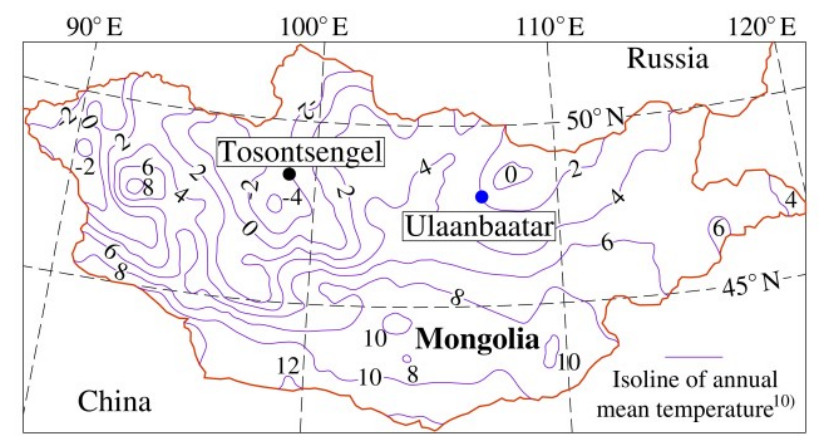

Fig.1 Location of the Tosontsengel hydropower plant 
(a)

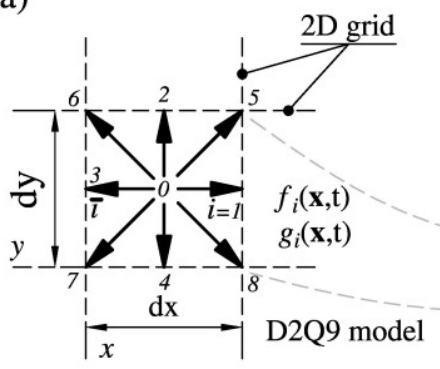

(b)

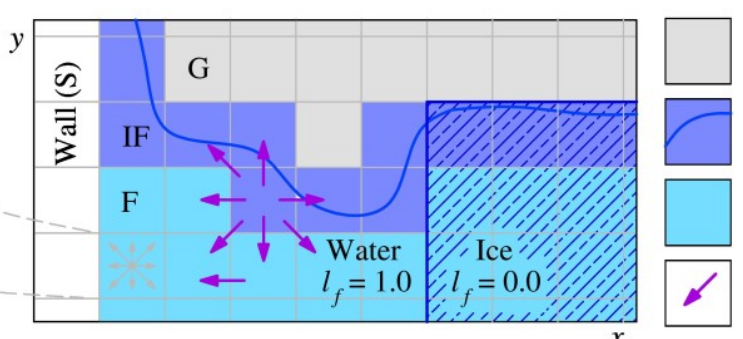

Air

(gas - G)

Free surface

(Interface - IF)

Water

(liquid - F)

Possible mass exchanges

Fig.2 D2Q9 lattice arrangement (a) and free surface representation with cell types (b)

which take into account the free surface flow and heat transport in the fluid flow. The phase changes of the water performed using the heat transport module are simulated using an enthalpy-based non-iterative approach $^{5), 6)}$. The free surface LBM as proposed by Thurey et $\mathrm{al}^{7)}$ is adapted and integrated with the heat transport module.

\section{NUMERICAL MODEL}

In this section, the basic concept and formulation of the proposed numerical model is described concisely. The model takes advantage of the features of the LBM over other conventional methods. For instance, the single phase approach for free surface flow is used to reduce the computational time. The immersed boundary method is used for modeling the boundary condition of the interface between the liquid and solid phases in the fluid flow.

\section{(1) LBM for free surface flow}

In the LBM, fluid flow is described by a finite set of distribution functions $f_{i}(\mathbf{x}, t)$ directed toward specified directions on the lattice, as shown in Fig.2 (a). The distribution functions of fluid flow in this model are integrated in time by using the discretized Boltzmann equation including BGK collision operator and the modification of immersed boundary ${ }^{8)}$

$$
\begin{aligned}
& f_{i}\left(\mathbf{x}+\mathbf{c}_{i} \Delta t, t+\Delta t\right)-f_{i}(\mathbf{x}, t)=-\frac{\Delta t(1-\beta)}{\tau_{t o t}} \\
& \times\left(f_{i}(\mathbf{x}, t)-f_{i}^{e q}(\mathbf{x}, t)\right)+\beta f_{i}^{m}(\mathbf{x}, t)+\Delta t F_{i},
\end{aligned}
$$

where $\mathbf{x}$ is the space vector, $\boldsymbol{c}_{i}$ is the discrete unit velocity in direction $i, \Delta t$ is the time step, and $\tau_{t o t}$ is the total relaxation time with respect to the addition of the lattice fluid viscosity and local eddy viscosity (the Smagorinsky model ${ }^{9}$ ). The modification parameter of the immersed boundary $\beta$ is defined by the liquid fraction value $l_{f}(\mathbf{x}, t)$. This has a value of 1 for water, 0 for ice, and between 0 and 1 for a liquid-solid interface, as shown in Fig. 2(b). An additional collision term $f_{i}^{m}$ models the liquid-solid interactions in terms of boundary condition at the interface between liquid and solid phases. This is calculated for cells that are par- tially or fully in the solid phase using the following equation:

$$
f_{i}^{m}(\mathbf{x}, t)=f_{\bar{i}}(\mathbf{x}, t)-f_{i}(\mathbf{x}, t)+f_{\bar{i}}^{e q}\left(\rho, \mathbf{u}_{s}\right)-f_{i}^{e q}(\rho, \mathbf{u}) .
$$

Here, $\mathbf{u}_{\mathrm{s}}$ is the velocity of the immersed body. This is set to 0 in the present study, i.e., ice is assumed to be stationary. A force term proposed by Guo et al. ${ }^{10)}$ is used for $F_{i}$ in Eq. (1) involving gravitational and buoyance forces. The equilibrium distribution function for fluid flow can be computed as

$$
f_{i}^{e q}=w_{i} \rho\left[1+\frac{\mathbf{c}_{i} \cdot \mathbf{u}}{c_{s}^{2}}+\frac{\left(\mathbf{c}_{i} \cdot \mathbf{u}\right)^{2}}{2 c_{s}^{4}}-\frac{\mathbf{u} \cdot \mathbf{u}}{2 c_{s}^{2}}\right],
$$

where $c_{S}$ is the speed of sound in the lattice form. The macroscopic fluid variables, density $\rho$, and velocity vector $\mathbf{u}$, are defined by the order of moments of the distribution functions as

$$
\rho=\sum_{i=0}^{8} f_{i}, \quad \rho \mathbf{u}=\sum_{i=0}^{8} \mathbf{c}_{i} f_{i}+\frac{\mathbf{F} \Delta t}{2} .
$$

As shown in Fig. 2(b), there are several types of cells, occupied by water, free surface, air, or solid walls ${ }^{11)}$. Free surface cells are called interface cells (IF) and the motion of an IF cell is tracked by changes in the volume fraction value of fluid in the cell. The volume fraction value is defined as the ratio of mass to density of the cell. Mass on the interface cell is estimated by the mass flux with neighboring fluid or interface cells using distribution functions. Depending on whether an IF cell is full or empty, further transformation of the IF cell is decided by value of the volume fraction. Transformation of an IF cell affects variables and the status of neighboring fluid or gas cells. More details are given in Rude and Thurey's work $^{7)}$. To satisfy the free surface condition, the following boundary conditions must be imposed on IF cells during the streaming process.

$$
f_{\bar{i}}^{\prime}(\mathbf{x}, t+\Delta t)=f_{i}^{e q}\left(\rho_{\mathrm{A}}, \mathbf{u}\right)+f_{\bar{i}}^{e q}\left(\rho_{\mathrm{A}}, \mathbf{u}\right)-f_{i}(\mathbf{x}, t),
$$

where $\rho_{\mathrm{A}}$ is the air density, which is directly connected to air pressure by the equation of state.

\section{(2) LBM for he at transport and phase changes}

After temporal calculation of the flow field, the heat transport must be computed using the thermal Boltzmann equation for temperature field, given by 

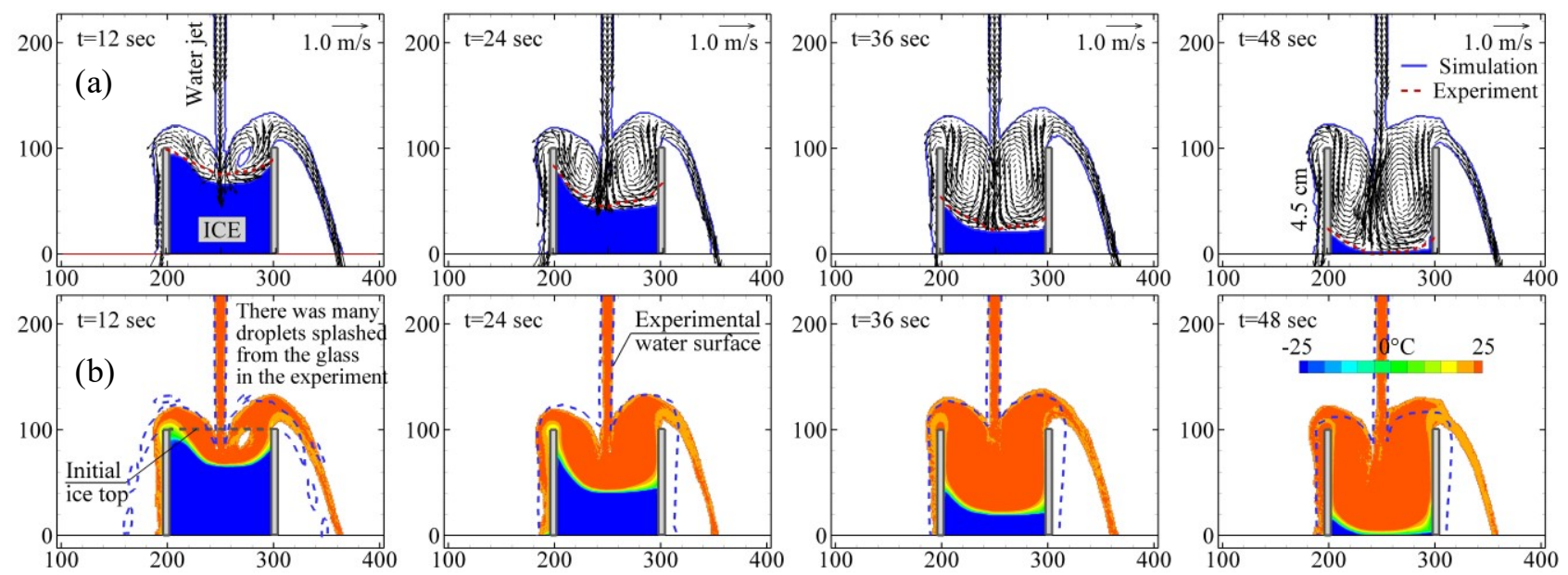

Fig.3 Temporal states of liquid-solid phases (a) and temperature fields during the ice melting by pouring water (b)

$$
\begin{gathered}
g_{i}\left(\mathbf{x}+\mathbf{c}_{i} \Delta t, t+\Delta t\right)-g_{i}(\mathbf{x}, t)=-\frac{g_{i}(\mathbf{x}, t)-g_{i}^{e q}(\mathbf{x}, t)}{\tau_{h}} \\
-w_{i} \frac{L_{h}}{c_{p}}\left(l_{f}(\mathbf{x}, t-\Delta t)-l_{f}(\mathbf{x}, t)\right),
\end{gathered}
$$

where $g_{i}(\mathbf{x}, t)$ is the distribution function for the temperature field, $\tau_{h}$ is the relaxation time with respect to the thermal diffusivity of water or ice, $L_{h}$ is the dimensionless latent heat, and $c_{p}$ is the specific heat capacity of water or ice. The equilibrium distribution function for the temperature field is given by

$$
g_{i}^{e q}=w_{i} \theta\left[1+\frac{\mathbf{c}_{i} \cdot \mathbf{u}}{c_{s}^{2}}\right] \text { with } \theta=\sum_{i=0}^{8} g_{i},
$$

where $\theta$ is the dimensionless temperature used for local enthalpy estimation using the enthalpy formula ${ }^{5}$. The liquid fraction value of a cell is calculated by linear interpolation of the local enthalpy value as

$$
l_{f}(x)=\left\{\begin{array}{cc}
1 & \text { for } E n>E n_{s}+L_{h}=E n_{l} \\
0 & \text { for } E n<E n_{s}=c_{p} \theta_{m e l t} . \\
\frac{E n-E n_{s}}{E n_{l}-E n_{s}} & \text { for } E n_{s} \leq E n \leq E n_{l}
\end{array}\right.
$$

In the free surface treatment of LBM, the distribution functions of the newly generated cells need to be reinitialized with equilibrium distribution functions using average variables of the surrounding fluid or IF cells ${ }^{7}$. The average variables were also used as the macroscopic variables for those cells ${ }^{6}$.

\section{NUMERICAL TEST}

The ice melting process was simulated using the proposed model in a closed domain and the obtained results compared with those reported by other studies $^{6}$. In the evaluation, we melted an ice cube by pouring water on it using 2D numerical simulation. The results were compared with a 3D laboratory experiment carried out using the same configuration so as to verify the numerical model. A glass with an ice cube was placed on a plate and water was poured

on the center of the top surface of ice. The ice cube size was $4.5 \times 4.5 \times 4.5 \mathrm{~cm}$. The temperature of the poured water was $30^{\circ} \mathrm{C}$, while the initial ice temperature was $-30^{\circ} \mathrm{C}$. The air temperature was taken as $25^{\circ} \mathrm{C}$ and a constant temperature boundary condition was applied at the inlet boundary and the free surface. In terms of heat transport, the walls and bottom surface were assumed to be adiabatic in the simulation. The bounce forward boundary condition ${ }^{11)}$, which reflects the distribution function at the wall like a mirror, was used to impose a slip boundary at the glass wall. Since it is difficult to capture the temperature distribution inside the ice experimentally, the evolution of the ice melting front against time was used as a metric to compare the numerical results. Because of the high temperature difference between the ice and the poured water, the ice melting process took over 1 minute. The temporal change in the melting front and the remaining ice area were monitored by processing the images recorded using a camera during the experiment. In Fig.3 (a), the variation in experimental and numerical melting front with free surface and velocity vector is shown at different time sequences. Experimental and numerical melting fronts are almost the same, as seen in Fig.3 (a). In the experiment, the water overflowed from the glass in all directions. However, in the simulation, the water overflowing from the left side is modeled as being attached to the side, while the water overflowing from the right side is computed as a weir flow as shown in Fig.3 (a). The melting front in the right side is more active than that in the left side in the simulation; this is also the case in the experiment. The heat transport at the left side was lower than that of the right side, as shown in Fig.3 (b). The ratio of the remaining ice area to the initial ice area for each of the cases is defined and compared in Fig.4. The melting rate of the experiment and simulation was nearly linear in comparison with the best-fit lines. The melting rate in the simulation was higher 


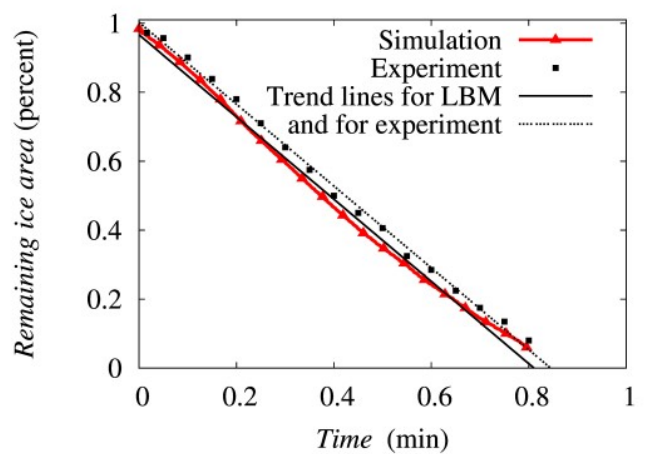

Fig.4 Experimentally and numerically determined remaining ice percentage

than that obtained from the experiment. The slight difference in the melting front evolution and the overflowing of water from the glass can be attributed to the dimensional difference. The temporal changes in the melting front and the flow of water over the side of glass show the asymmetric properties in the simulation. The asymmetric melting front obviously leads to asymmetric water overflow. Another reason for the asymmetric flow might be that the surface tension of the water is not considered in the free surface boundary condition. It seems that the surface tension affects the water overflow in the lab experiment. The asymmetry of the melting front also can be related to the free surface boundary condition. However, the overall predictions of the numerical model were in reasonable agreement with the experiment.

\section{NUMERICAL APPLICATION}

\section{(1) Problem statement}

In the winter of 2007, a year after its commissioning, the Tosontsengel hydropower plant experienced ice jam and ice ridge formation in the downstream area between the outlet structure and a bridge that is located around $130 \mathrm{~m}$ downstream of the plant. An engineer working in the plant recalls, "Power production did not meet the expected operation plan and the efficiency was lower. In addition, ice heavily affected the bridge pier as an excess load. That year, workers in the plant suspected that fluctuations in the water discharge and the low speed of water in the outlet created the downstream ice problem." The next year, they experimented by passing water downstream through the turbine without operating it. In other words, the water discharge through the turbine was the same as the river flow rate. Consequently, the ice jam reduced greatly but small ice ridges were still observed downstream. Since then, the plant authorities decided to use this mechanism in the winter. When passing water through a stationary turbine, the water surface is not enclosed by ice for a distance of 4 to $5 \mathrm{~m}$ from the outlet. This is termed open water, and it exists during the winter. No research has been

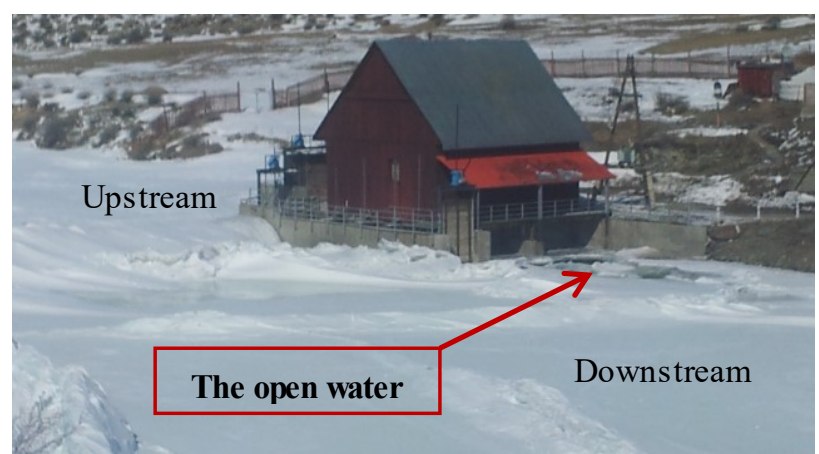

Fig.5 The Tosontsengel hydropower plant, March 24, 2014.

conducted on a way to deal with the ice and operate the plant during the winter. However, workers are still seeking a way to produce power in the winter. In this study, we simulate the process of open water formation (see Fig.5) in the downstream. This occurs due to the release of warm water from the upstream through the turbine. It provides the possibility of applying a numerical model to the ice phenomena in hydropower plants. Absence of field data and computational limitations make it difficult to model the ice problem on a large scale.

\section{(2) Nume rical results}

Fig.6 shows a longitudinal cross-section of the Tosontsengel hydropower plant including the computational domain. The computational domain does not include the upstream and the spiral case of the turbine. Hence, the inlet boundary of the computational domain is at the beginning of the turbine tailrace. The domain extends to $32 \mathrm{~m}$ downstream. We use a grid spacing $\Delta \mathrm{x}=0.04 \mathrm{~m}$ and a time step $\Delta \mathrm{t}=4.11 \times 10^{-4}$ $\mathrm{s}$. We assume that the downstream of the plant is fully covered with ice of thickness $0.3 \mathrm{~m}$ and the wicket gates of the turbine are closed initially. The temperature of the ice cover is taken to be $-10^{\circ} \mathrm{C}$ in all simulations. The simulation starts with the opening of the wicket gates. At the inlet boundary, velocity and constant temperature conditions are imposed for the fluid flow and heat transport modules, respectively. Velocities at the inlet boundary are approximated from the capacity of the small turbine because of lack of winter flow data. At the outlet boundary, the second order extrapolation boundary condition ${ }^{12)}$ was applied to the fluid flow and the heat transport modules. A typical bounce back boundary condition is used for the concrete wall and channel bed as a no-slip boundary condition. As observed in the field, there is a continuous ice cover through the downstream area. Hence, the ice cover is assumed to be stationary and there are no crushing phenomena in the simulation. In order to understand the formation of open water in the ice covered downstream, three different water temperatures, $2^{\circ} \mathrm{C}, 4^{\circ} \mathrm{C}$, and $8^{\circ} \mathrm{C}$, are 


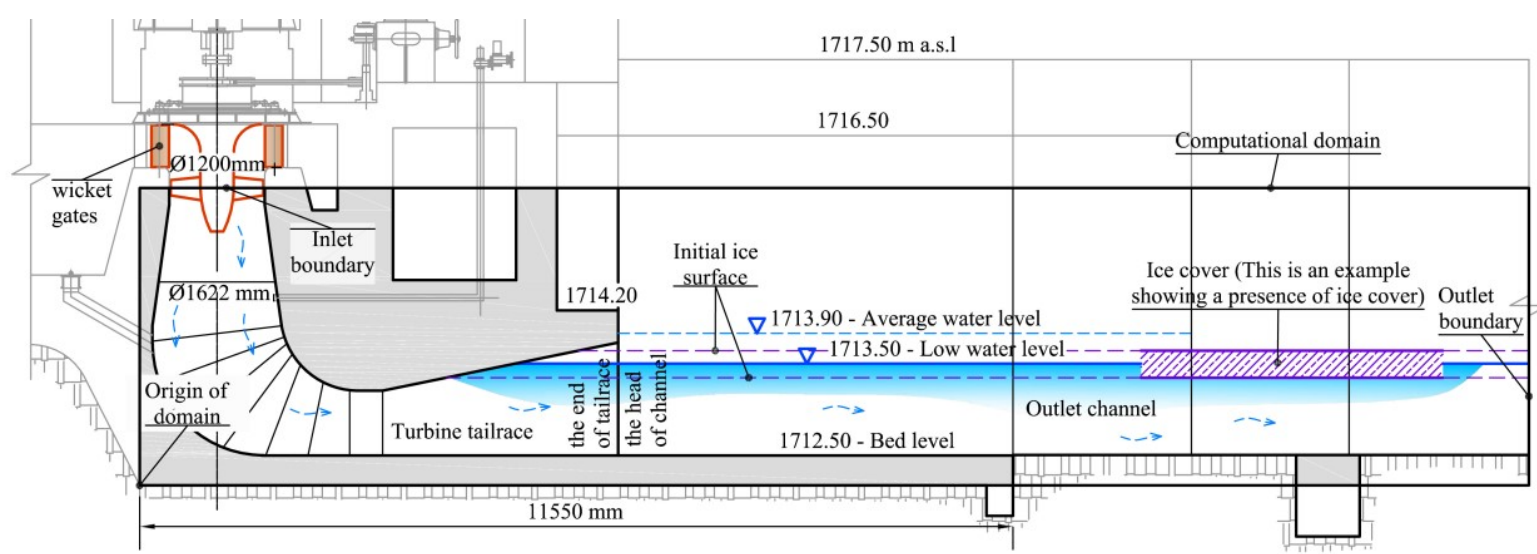

Fig.6 A longitudinal cross-section of the Tosontsengel hydropower plant and computational domain with boundary conditions. considered as cases for the low discharge water in the simulations. As shown in Fig. 7, in case 1, the ice area evolution was stable except for an initial drop that could have been caused by initial pulses of inflow. Melting should have started near the end of the tailrace, where the velocity is the highest. However, open water was not formed during more than 10 minutes of simulation, as seen in Fig.8 (a), and eventually the system went into equilibrium. This is because of the low heat transfer rate between ice and water at the bottom of the ice cover. Melting or freezing of the ice cover happens in the other two cases. As the temperature is lower, the melting rate was lower for case 2 in comparison with case 3 . With the same hydraulic conditions, open water was created at different times; this is seen as a transition of the remaining ice area in Fig.7. After the open water is formed, freezing occurs in case 2 , because of the water overflow and the low discharge of water under the ice cover. In case 3 , the ice area gradually decreases because the temperature of water is sufficient to melt ice on and under the ice cover. This is shown in Fig. 7 and Fig.8 (c). The air temperature is shown in Fig. 8 (b) and is the same for all cases. As shown in Fig. 8, the length of the open water was 0.18 $\mathrm{m}$ for case 2 and $0.4 \mathrm{~m}$ for case 3 . The open water in case 3 was further extended to $2.08 \mathrm{~m}$ in length at 13 minutes and $4.0 \mathrm{~m}$ at 23 minutes.

The end of the tailrace is a special region, where the temperature of water is almost the same as that of the initial boundary. This is where the heat exchange between water and ice starts. The flow is also intensive and is directed at the ice cover. The hydraulic and thermal conditions that exist in this region form the basic elements for the formation of open water. The open water formation mechanism can be described by following process. Initially, ice warming and melting processes take place at the end of the tailrace. This one sided melting process ends at the initial opening of the ice cover. The initial opening can appear adjacent to or near the concrete wall, depending on the geometry of the tailrace. Melting at more than two sides can occur for the ice cover, if water flows out through the initial opening and flows over the ice surface. The melting rate depends on the water temperature and the discharge. If the discharge and temperature are sufficient to melt ice, the open water will be extended. This extension process takes place until the equilibrium state of the open water is reached. In the other case of temperature and discharge, ice cover will be extended in depth and eventually the downstream will be covered by ice again.

Hydraulically, the water flowing at the end of the tailrace tends to flow in a direction upward to the free surface. It then flows toward the downstream channel. The flow at the end of tailrace, as pointed on Fig.8 (a), is intensified when the downstream is covered with the ice and open water exists in the head of downstream channel. In other words, the ice covered channel acts against the water coming from the turbine, like the flow through a conduit under pressurized conditions. The open water is under low pressure and interacts with the air; hence, water can easily flow in an upward direction after leaving the tailrace, as seen in Fig. 8 (b) and (c). Therefore open water is maintained during winter.

The fact is that when the temperature is lower than $2^{\circ} \mathrm{C}$ and the discharge of the released water is approximately the maximum capacity of the turbine, the downstream ice cover does not melt. This implies that

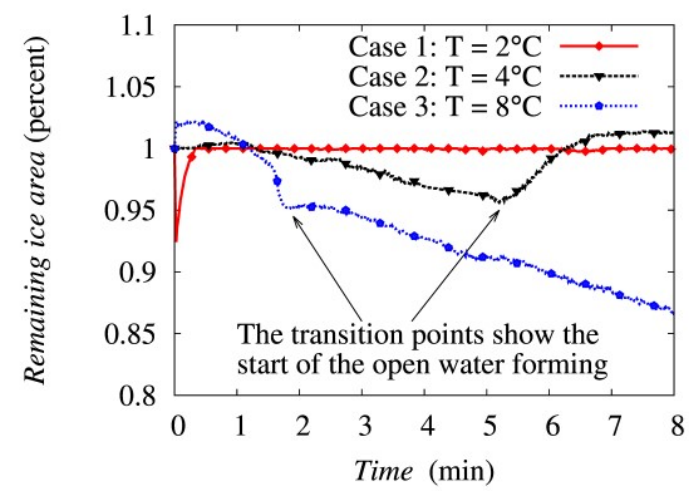

Fig.7 Numerically determined remaining ice area of the three temperature cases. 
(a)

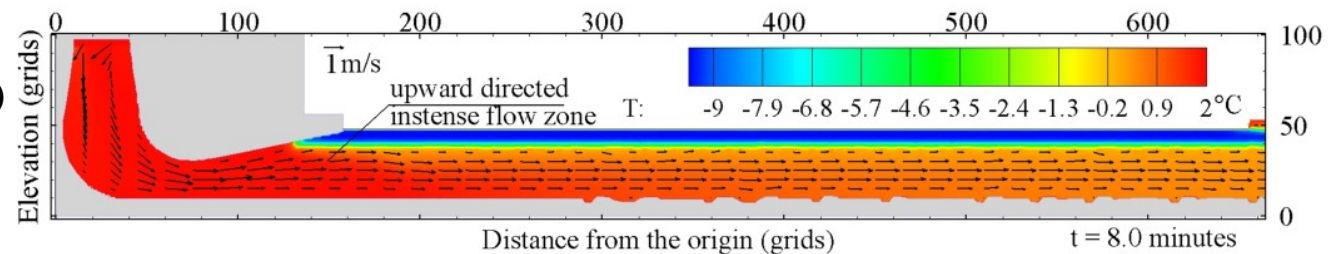

(b)
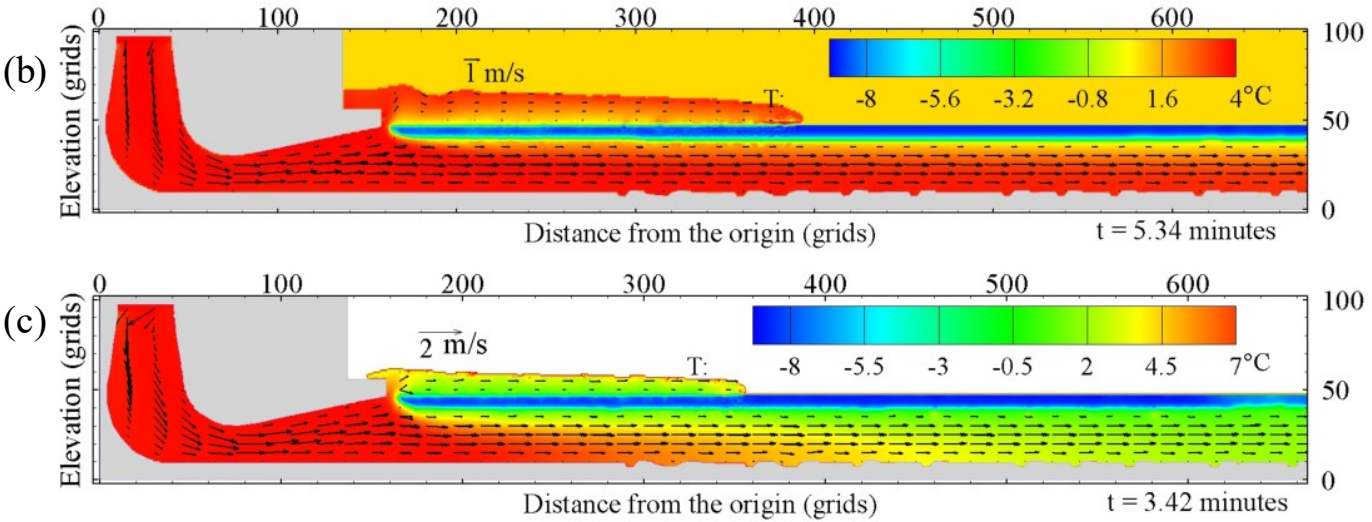

Fig.8 Numerically defined temperature field and flow field at different times and cases: (a) case 1, (b) case 2 and (c) case 3 .

when water at a temperature near $2^{\circ} \mathrm{C}$ is flowing through the downstream channel and there exists open water in the downstream, ice will not melt or extend if there is no overflow above the ice cover from the open water. Based on the flow characteristics obtained from the above simulation, if the flow direction in the head of the downstream channel is adjusted through the channel, it is possible to control downstream ice phenomena for different discharge conditions.

\section{CONCLUSIONS}

The LBM modeling for liquid-solid phase changes of water in a free surface flow was presented. The proposed model was tested by simulating the melting of ice by pouring water. The result of the numerical test was in good agreement with the experiment conducted using the same configuration. The proposed model was applied to form open water in the downstream channel of the Tosontsengel hydropower plant. Results indicate that if the discharge of water flowing through the turbine is constant, the downstream ice condition will be steady in general. If the water temperature goes up by about $2^{\circ} \mathrm{C}$, ice can be affected and depending on the discharge, ice melting or extension will take place. The proposed model can be used for studying the ice phenomena in the downstream of hydropower plants for a short period of about two days. The results could be used to arrive at an operation plan to control ice in the winter. To analyze ice phenomena in more detail, the model must be extended to cover ice cracking due to volume extension of ice. The immersed boundary method employed in the proposed model is the basis of the floating ice in free surface flow. A floating ice simulation using this model is under development.

\section{REFERENCES}

1) Mongolian sustainable energy sector development strategy plan, 2002

2) Boldbaatar, B.: Assessment of future hydropower plant investment in Mongolia, Master thesis, Seoul National University., pp. 25-28, 2013.

3) Ecology and social-economy study on the Ider river basin, Mongolian Water Forum, 2013

4) Gebre, S., Alfredsen, K., Lia, L., Stickler, M. and Tesaler, E. : Review of ice effect on hydropower systems, J. Cold. Reg. Eng, No. 27(4), pp. 196-222, 2013.

5) Huber, C., Parmigiani, A., Chopard, B., Manga, M. and Batchmann, O.: Lattice Boltzmann model for melting with natural convection, Int. J. Heat and Fluid flow, No. 29(5), pp. 1469-1480, 2008.

6) Ayurzana, B. and Hosoy amada, T.: Phase change simulations of water near its density inversion point by Lattice Boltzmann method, Proc. the 23rd Int. Sym. on Ice, 2016.

7) Körner, C., Thies, M., Hofmann, T., Thürey, N. and Rüde, U.: Lattice Boltzmann model for free surface flow for modeling foaming. J. Stat. Phys., No. 121(1-2), pp. 179-196, 2005.

8) Noble, D.R. and Torczynski, J.R.: A lattice-Boltzmann method for partially saturated computational cells. Int. J. Modern Phy. C, No. 9(08), pp.1189-1201, 1998.

9) Hou, S., Sterling, J., Chen, S. and Doolen, G. D.: A lattice Boltzmann Subbgrid Model for High Reynolds Number Flows. Pattern Formation and Lattice Gas Automata, No. 6, p. 149. 1994.

10) Guo, Z., Zheng, C. and Shi, B.: Discrete lattice effects on the forcing term in the lattice Boltzmann method, Physical Review E, No. 65(4), pp. 046308, 2002.

11) Ayurzana, B., Hosoyamada, T. and Nasanbayar, N.: Hydraulics application of the Free-surface Lattice Boltzmann method, Proc. IFOST-2016, No. 1(4), 2016.

12) Alamyane, A.A. and Mohamad, A.A.: Simulation of forced convection in a channel with extended surfaces by the lattice Boltzmann method. Com \& Math with Applications, 59(7), pp.2421-2430, 2010.

(Received September 30, 2016) 